\title{
BRAFV600E mutation in hairy cell leukaemia a single center descriptive cross sectional study
}

\begin{abstract}
Introduction: Hairy Cell Leukaemia (HCL) is a rare, clonal, chronic lymphoproliferative disorder characterized by Pancytopenia with moderate to massive splenomegaly. Peripheral blood and bone marrow smears show lymphocytes with abundant cytoplasm and hair-like processes. The morphological, cytochemical and immunophenotypic features of HCL classic can mimic HCL variant, which can cause diagnostic difficulties. The acquired V600E mutation of the BRAF gene has been described as a molecular marker for HCL classic and this mutation is mostly not detected in other chronic B cell Lymphoproliferative disorders and in HCL v. Keeping in view the significance of BRAF V600E mutation in $\mathrm{HCL}$, the present study was designed with an aim to study the frequency of BRAF V600E mutation in HCL, which would be of help in differentiating it from HCL $\mathrm{v}$ and also in establishing diagnosis, determining treatment protocols and prognosis.
\end{abstract}

Methods: A total of 45 patients suspected to have HCL were included in this study. Blood complete picture, bone marrow examination (aspiration and trephine biopsy), cytochemical staining (TRAP), immunophenotyping and BRAF V600E mutation (on bone marrow aspirate samples) of the patients suspected to have HCL was done. BRAF V600E Mutation was performed by PCR and directs DNA Sanger Sequencing.

Results: BRAF V600E mutation was detected in $36(80 \%)$ cases and it was not detected in $9(20 \%)$ of $\mathrm{HCL}$

Conclusion: The BRAF V600E mutation was present in majority of HCL cases and it is absent in HCL $\mathrm{v}$ cases. However a minority of HCL cases were without this mutation. The presence of BRAF V600E mutation has relevant implications for the pathogenesis, diagnosis and targeted therapy of HCL.

Keywords: BRAF V600E mutation, sanger sequencing, monocytopenia, CBC, CLL, B-cell lymphoproliferative, reticulin fibrosis
Volume 5 Issue 4 - 2017

\author{
Ayesha Khurshid, Saleem Ahmed Khan, \\ Chaudhry Altaf Hussain, Ahsan Hussain, \\ Hamid Saeed Malik, Parvez Ahmed \\ Department of Haematology, Armed Forces Institute of \\ Pathology, Pakistan
}

\section{Correspondence: Ayesha Khurshid, Pathologist} (Haematologist), Department of Haematology, Armed Forces Institute of Pathology, CMH Road, Rawalpindi (46000), Pakistan, Tel+923322222485, Email ayeshafaisal82@yahoo.com

Received: August 08, 2017 | Published: November 01, 2017
Abbreviations: HCL, hairy cell leukaemia; HCLv, hairy cell leukaemia variant; TRAP, tartrate-resistant acid phosphatase; ACP, acid phosphatase; DLC, differential leukocyte count; TLC, total leukocyte count; IHC, Immuno Histo Chemistry; EDTA, ethylene diamine tetra acetic acid

\section{Introduction}

HCL is a B-cell chronic lymphoproliferative disorder which is uncommon but has distinct features characterized by indolent course, splenomegaly, cytopenias usually affecting two or three lineages and absolute monocytopenia. Neoplastic lymphoid cells with hairy cells are present in the peripheral blood, bone marrow, spleen, liver and associated with reticulin fibrosis of bone marrow. ${ }^{1}$ HCL was first described as a distinct clinico-pathological entity in 1958 by Bouroncle et al. ${ }^{2}$ at Ohio State University College of Medicine. ${ }^{2}$ It account for $2 \%$ of all leukemias and $8 \%$ of all mature $\mathrm{B}$ and $\mathrm{T}$ cell lymphoproliferative diseases. It affects middle-aged men more commonly than women; the male: female ratio is $4.5: 1$ and median age at diagnosis is 50years. ${ }^{3}$ Typical clinical features are splenomegaly with or without hepatomegaly, pallor, fatigue, weight loss, infections, hemorrhage and rarely lymphadenopathy. Its most common presentation is pancytopenia with moderate to massive splenomegaly. ${ }^{4}$ Lymphadenopathy though rare is associated with more aggressive disease progression. ${ }^{5}$ In $10 \%$ of cases, the disease is asymptomatic.

Blood complete picture (CBC) shows low neutrophil count, monocytopenia and some cases present as moderate to severe pancytopenia. ${ }^{6}$ Peripheral blood smear reveals hairy cells with characteristic morphological features: large lymphoid cells, $10-15 \mu \mathrm{m}$ in diameter, with low N/C ratio, central or eccentric position of round, oval or indented nuclei, reticular or netlike chromatin pattern, with indistinct or absent nucleoli. The pale blue cytoplasm presents fine, hair-like projections or ruffled borders. Bone marrow aspiration film is usually diluted or a dry tap which may show the presence of few hairy cells. Bone marrow trephine biopsy is more informative and reveals characteristic pattern of infiltration, with cells appearing separated because of their abundant clear cytoplasm. In almost all cases of HCL reticulin stain shows moderate to marked increase in reticulin fibers. ${ }^{7}$ In cytochemical staining the hairy cells stain positively for tartrate resistant acid phosphatase (TRAP) ${ }^{8}$ Flow cytometry studies are now routinely done on either peripheral blood or bone marrow aspirate samples. Pan B cell markers, such as CD19, CD20 and CD22, are always positive. The classic HCL cells are positive for CD11c, CD25 and $\mathrm{CD} 103 .{ }^{7} \mathrm{HCL}$ variant is identified by morphological features (medium to large size lymphocytes, prominent nucleoli), leukocytosis at diagnosis, absence of monocytopenia and bone marrow fibrosis and lack of typical hairy cell leukaemia phenotype on flow cytometry. ${ }^{9}$ 
Recently, the acquired V600E mutation of the BRAF gene has been described as a specific molecular marker for classical HCL by Tiacci et al. ${ }^{10}$ This mutation is usually not detected in other chronic B cell lymphoproliferative disorders and in HCL variant with similar clinical and morphological features. ${ }^{10}$ However this mutation is sometimes present in metastatic melanomas, thyroid carcinoma and colon carcinomas. ${ }^{11}$ The BRAF V600E has been reported in other B cell malignancies (CLL, myeloma, NHL, LPL) albeit at a much lower frequency. BRAF is a human gene that makes a protein called B-Raf. The gene is also referred to as proto-oncogene BRAF. The BRAF V600E mutation is present in all patients with HCL and that, in combination with clinical and morphologic features, represents a reliable molecular marker for the laboratory diagnosis of this mature B-cell neoplasm.

The pathogenesis of HCL is not well understood. However BRAF represents the most frequently mutated gene encoding protein kinase in HCL and some other human cancers. ${ }^{8}$ This mutation is detected by PCR and Sanger Sequencing of BRAF exon-15. It revealed thiamine to adenine transversion occurring at position 1860 of the BRAF messenger RNA, resulting in substitution of Glutamic acid for valine, at position 600 of BRAF protein. As the mutation appeared as double peak, containing both thiamine and adenine, it indicates a heterozygous lesion in BRAF resulting in the BRAF V600E variant protein (Figure 1). The fact that this mutation is found in virtually all cases of classic HCL suggests that it is a key underlying genetic defect in the pathogenesis of this leukaemia. ${ }^{11}$ Arcaini et al. ${ }^{12}$ studied that this mutation is the genetic hallmark of this leukaemia and is present in nearly all the patients of HCL. ${ }^{12}$ The high frequency of BRAF V600E mutation in HCL classic and lack of such mutation in other B-cell malignancies indicates that this mutation represents a disease-defining mutation in HCL. ${ }^{13}$ However Xi et al..$^{14}$ and few other authors have now reported that BRAF mutation may not be present in all the HCL cases. ${ }^{14}$ We undertook this study in our tertiary care referral center to explore the frequency of BRAF V600E mutation in our patients and its role in the diagnosis of HCL.

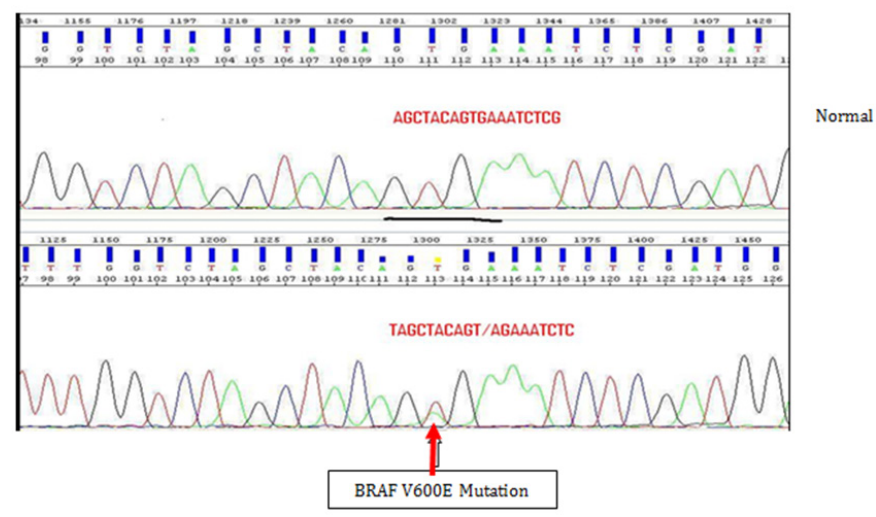

Figure I BRAFV600E mutation by sanger sequencing.

\section{Materials and methods}

This study was carried out in the department of Hematology, Armed Forces Institute of Pathology Rawalpindi, Pakistan. It was a descriptive, cross sectional study. The study was completed over a period of 1year, from 15 Oct 2015 to 14 Oct 2016. A total of 45 patients suspected to have HCL were included in this study. Sampling technique was non-probability consecutive sampling. Samples from patients of HCL classic and variant on initial workup of either gender or age were included. Patients of other lymphoproliferative disorders were excluded from the study. All subjects fulfilling the inclusion criteria were elaborately apprised about the study to obtain their informed consent. Patient particulars and detail laboratory and clinical findings of each patient were endorsed in a proforma for data analysis. $2.5 \mathrm{ml}$ venous blood in ethylene diamine tetra acetic acid (EDTA) was collected and blood complete count was performed by Sysmex KX 21 automated hematology analyzer. Blood smear was examined for DLC and lymphocyte morphology. Bone Marrow examination (aspiration and trephine biopsy) and cytochemical staining (ACP and TRAP) was done. Flow cytometry was done on bone marrow aspirate samples of the patients suspected to have HCL using pan B markers i.e., CD19, CD20 and hairy cell leukemia specific markers i.e., CD 103, CD11c and $\mathrm{CD} 25$.

\section{DNA sampling and extraction}

$2-4 \mathrm{ml}$ of bone marrow aspirate or peripheral blood was collected in EDTA and DNA extraction was done by $10 \%$ Chelex. Amplification was carried out by using following primers, 5'-TACCTAAACTCTTCATAATGCTTGC-3' and 5'-GTAACTCAGCAGCATCTCAGGG-3'. The BRAF exon 15 Primers were designed according to Tiacci et al. ${ }^{10}$ and were manufactured by Biotech. The reaction mixture included 1x PCR buffer, $2.5 \mathrm{mM} \mathrm{MgCl}_{2}, 10 \mathrm{pM}$ primers, $100 \mu \mathrm{M}$ of dNTPs, 0.5 units of Taq DNA polymerase (Invitrogen), $2 \mu \mathrm{g}$ of DNA and molecular biology grade water in total $25 \mu 1$ volume. PCR conditions include 30 Cycles of $94^{\circ} \mathrm{C}$ for $30 \mathrm{sec}, 60^{\circ} \mathrm{C}$ for $1 \mathrm{~min}, 72^{\circ} \mathrm{C}$ for $90 \mathrm{sec}$ and $72^{\circ} \mathrm{C}$ for 3mins in Gene Amp Thermal Cycler 9700 (Applied Biosystems USA). Amplified product was analyzed on $6 \%$ polyacrylamide gel followed by silver staining to check the quality of DNA. Prior to sequencing, the amplified product was purified by the PureLink PCR Purification Kit, (Invitrogen, Carlsbad, CA, USA) to remove the nonspecific amplification products, primer-dimers and unused dNTPs. The sequencing was carried out by Sanger's dideoxy chain termination method using the BigDye Terminator sequencing kit v3.1 (Applied Biosystems, Foster City, CA, USA). PCR conditions include 25 Cycles of $96^{\circ} \mathrm{C}$ for $10 \mathrm{sec}, 50^{\circ} \mathrm{C}$ for $5 \mathrm{sec}, 60^{\circ} \mathrm{C}$ for 4 minutes. Amplified fragments were purified by Agencourt CleanSeq (Beckman Coulter USA). Purified product was analyzed on Genetic Analyzer 3130 (Applied Biosystem USA) and data was analyzed on Sequencing Analysis software v 5.3.1 (Applied Biosystem USA) (Figure1).

\section{Results}

A total of 45 subjects were enrolled in the study. Out of the $45 \mathrm{HCL}$ patients, 39 were males $(86 \%)$ and 06 were females $(14 \%)$. Male to female ratio was 6:1. Age of patients ranged between 27 to 67 years. Median age in the HCL patients was 50years. Majority of the HCL patients were found in the fourth and fifth decade of life. Most common symptom was fever, pallor and abdominal distension. Splenomegaly was found in all patients. The median spleen enlargement below costal margin in the HCL was $8 \mathrm{~cm}$. Minimum spleen enlargement was $2 \mathrm{~cm}$ and maximum was $21 \mathrm{~cm}$. The median $\mathrm{WBC}$ in the HCL was $3.0 \times 10^{9} / \mathrm{L}$. Minimum $\mathrm{WBC}$ was $1.17 \times 10^{9} / \mathrm{L}$ and maximum was $186.2 \times 10^{9} / \mathrm{L}$. Only two patients had very high WBC count. The median $\mathrm{Hb}$ in the $\mathrm{HCL}$ was $9.5 \mathrm{~g} / \mathrm{dl}$. Minimum $\mathrm{Hb}$ was $5.4 \mathrm{gm} / \mathrm{dl}$ and maximum was $13.4 \mathrm{~g} / \mathrm{dl}$. Anemia was present in $32(71 \%)$ patients at the time of presentation. The median platelet count in the HCL was $55 \times 10^{9} / \mathrm{L}$. Minimum platelet count was $13 \times 10^{9} / \mathrm{L}$ and maximum was $149 \times 10^{9} / \mathrm{L}$. 
All patients had variable number of hairy cell in the peripheral blood film. The median percentage of hairy lymphocytes in the HCL was $50 \%$. Minimum percentage of hairy lymphocytes was $5 \%$ and maximum was $88 \%$. In two cases the hairy projection was less prominent with prominent nucleoli and high nuclear-cytoplasmic (N/C) ratio. These two cases may represent HCL variant. Monocytopenia was present in $40(88 \%)$ patients. Bone marrow aspiration was diluted in $32(71 \%)$ patients. Variable numbers of hairy cells were seen in the bone marrow aspirates of all patients. TRAP positivity was seen $35(78 \%)$, weak positivity was seen in $2(4 \%)$ cases and $8(18 \%)$ patients were TRAP negative. Trephine biopsy sections showed abnormal infiltrate in all the cases. The typical fried egg appearance (peri-nuclear halo or clearing) was seen in $43(95.5 \%)$ patients. The remaining $2(4.5 \%)$ cases showed interstitial abnormal lymphoid infiltrate. Focal and Diffuse fibrosis was seen in $42(93 \%)$ cases, ranging from grade I to IV by reticulin staining and unremarkable in $3(7 \%)$ cases. CD25, CD103, CD11c and CD19 positivity was seen in $40(89 \%)$ patients by flow cytometry. 5(11\%) patients were negative for CD25. BRAF mutation was positive in $36(80 \%)$ cases and was not detected in $9(20 \%)$ cases. The main findings of all cases are summarized in Table 1. BRAF V600E mutation in HCL patients was statistically significant (P value $<0.04$ ) as compared to patients with HCL Variant.

\section{Discussion}

HCL is strongly associated with BRAF V600E mutation. However it is usually not present in HCL $v$ and splenic marginal zone lymphoma with villous lymphocytes. This mutation is the genetic hallmark of HCL and is present in nearly all the patients of HCL as reported by previous studies. ${ }^{10,11}$ However it is also present with a lower frequency in some other B cell malignancies (CLL, Myeloma, NHL, LPL) and in papillary thyroid carcinoma, colorectal cancer, melanoma and nonsmall-cell lung cancer. Xi et al. ${ }^{14}$ identified some patients of HCL with no BRAF V600E mutation. Out of his 53 cases 11 patients were lacking BRAFV600E mutation. ${ }^{14}$ This generated a lot of interest for other researcher to explore the status of BRAFV600E mutation in their HCL patients. As we also carried out our study to find out frequency of BRAFV600E Mutation in HCL.

No study is published so far on the molecular analysis of HCL patients in Pakistan. Keeping in view the significance of BRAF V600E mutation in HCL, the present study was designed with an aim to study the frequency of BRAF V600E mutation in HCL, which would be of help in diagnosis, determining treatment protocols and prognosis. BRAF inhibitors (vemurafenib) can be used as targeted therapy in HCL patients especially in refractory HCL with promising results. ${ }^{15-17}$ Inhibitors of BRAFV600E have provided responses in patients who are resistant to standard therapy. Vemurafenib also showed excellent activity in recurrent, relapsed and refractory HCL patients with rapidly decreased splenomegaly, increased platelet counts and normalization of hemoglobin and granulocyte counts. The optimal dosing and duration of treatment using vemurafenib is unknown. A multicentre, phase II study in the treatment of refractory HCL, of vemurafenib is ongoing (NCT01711632).$^{18}$ Our study showed that out of 45 HCL patients, 39 were males $(86 \%)$ and 06 were females $(14 \%)$. The male predominance in our study population is comparable with international studies. Majority of studies showed predominance of males. However in our study male predominance is even more $(6: 1)$. In a study reported from Tiacci et al., ${ }^{10}$ out of total 47 patients $37(79 \%)$ were males and 10(21\%) were females. Male to female ratio was 3.7:1 However Arcaini et al., ${ }^{12}$ in their study has shown that out of total 62 patients $52(84 \%)$ were males and $10(16 \%)$ were females. Male to female ratio was 5.2:1.
The age of the patients with HCL in our study ranged from 27 to 67 years with median age was 50years. Tiacci et al. ${ }^{10}$ in their study has shown that age of patients with HCL ranged from 33 to 80 years with a median age of 56 years. In another study conducted in Italy by Arcaini et al. ${ }^{12}$ age of patients with HCL ranged from 36 to 84 years with a median age of 60years which also matches our study. In our population majority of HCL patients are seen at comparatively younger age group, probably because Pakistan generally harbours younger population or there might be some biological variation of the disease in the sub-continent. Median WBC count in the HCL in our study was $3.0 \times 10^{9} / \mathrm{L}$. Minimum WBC was $1.17 \times 10^{9} / \mathrm{L}$ and maximum was $186.2 \times 10^{9} / \mathrm{L}$. Tiacci et al. $^{10}$ showed median WBC of $6.716 \times 10^{9} / \mathrm{L}$. Minimum WBC was $1.30 \times 10^{9} / 1$ and maximum was 51.0x $10^{9} / 1$, which is lower as compare to our study. A study done by Arcaini et al. ${ }^{12}$ showed that patients with $\mathrm{WBC}$ of $<4 \times 10^{9} / \mathrm{L}$ in 39 $(63 \%)$ and $>10 \times 10^{9} / \mathrm{L}$ in $5(8 \%)$ and in our study WBC of $<4 \times 10^{9} / \mathrm{L}$ was in $28(62 \%)$ and $>10 \times 10^{9} / \mathrm{L}$ was in $17(38 \%)$ which is comparable to study by Arcaini et al. ${ }^{12}$ The principal observation of our study was the results of DNA Sanger Sequencing, which showed BRAF V600E positivity in $36(80 \%)$ HCL classic patients, however; this mutation was not found in $9(20 \%)$ patients defined phenotypically as HCL.

Out of total $9(20 \%)$ patients which were negative for BRAF V600E mutation, 2 cases had very high TLC count i.e., 186x $10^{9} / \mathrm{L}$ and $65.09 \times 10^{9} / \mathrm{L}$ along with bicytopenia and massive splenomegaly but no monocytopenia. The hairy lymphocytes had less shaggy margins, fibrosis was unremarkable and TRAP was negative. Immunophenotyping revealed absence of CD 25 which is the specific marker of HCL c. So these were HCL v patients, lacking mutation. Among rest of 7 patients showing no BRAFV600E mutation, 3 were negative and 4 were positive for CD 25 in immunophenotyping. All 7 had splenomegaly ranging from 2 to $21 \mathrm{~cm}$ and pancytopenia along with monocytopenia except one case. Different percentages of hairy lymphocytes were present in the PB ranging from $20 \%$ to $80 \%$. Reticulin fibrosis was increased from grade I to IV except in one case in which reticulin fibrosis was unremarkable. In this case monocytopenia and CD25 were also absent. May be this case was also HCL v. The other two cases in which CD25 was absent have also not detected BRAFV600E mutation may be due to diluted marrow with low tumour cell burden or may be these cases were also HCL v. No control PCR was used to determine the quality of DNA extracted. Absence of the BRAF V600E in some cases may reflect poor quality DNA.

The other 4 cases in which CD25 was positive but BRAF V600E mutation was negative, may harbor a non-exon 15 mutations or it was falsely negative. Therefore in these 4 cases BRAFV600E mutation could have been detected if we had more sensitive techniques i.e., allele-specific polymerase chain reaction or next generation sequencing. A more recently published study Boyd et al. ${ }^{19}$ reported $100 \%$ of $48 \mathrm{HCL}$ classic samples to contain BRAF V600E mutation ${ }^{19}$ and also Tiacci et al. ${ }^{10}$ and Arcaini et al. ${ }^{12}$ showed $100 \%$ positivity of BRAFV600E mutation for HCL, whereas our study showed $80 \%$ positivity of BRAFV600E mutation for HCL. These results were comparatively lower to Boyd et al., ${ }^{19}$ Tiacci et al. ${ }^{10}$ and Arcaini et al. ${ }^{12}$ but comparable with $\mathrm{Xi}$ et al. ${ }^{14}$ which showed that BRAF was mutated in $42(79 \%)$ of HCL classic patients.

There are various postulations regarding BRAF V600E negativity in some HCL cases. Some could be truly wild type and some may lack the specific BRAF V600E mutation and instead harbor a non-exon 15 mutation or MAP2K1 mutations. ${ }^{20}$ The BRAF V600E mutation status may also be falsely negative due to low tumor cell burden and 
use of assays with limited analytical sensitivity. ${ }^{21}$ High resolution melting analysis (HRM) is a new and very sensitive technique for the detection of BRAF V600E mutation in cases where tumor burden is $<30 \%$. Blombery et al. ${ }^{9}$ studied that out of the 42 samples from patients with HCL, BRAF V600E mutation was detected by HRM in all cases but only 38 samples were positive for mutation by Sanger sequencing. It is likely that these samples contained the BRAF V600E mutation but it was below the sensitivity of Sanger sequencing.

Andrulis et al. ${ }^{22}$ studied application of BRAF V600E mutation specific antibody by Immunohistochemistry (IHC) to differentiate between HCL classic and HCL mimics (HCL variant and SMZL). He studied 30cases of HCL, all were positive for BRAF V600E mutation specific antibody (by IHC), however only 28 cases were positive for BRAF V600E mutation by Sanger Sequencing. Two cases of HCL that were positive for BRAF V600E by IHC lacked the mutation by DNA Sanger sequencing. Both cases, tumor burden was below the detection limit of Sanger sequencing. This suggests that in cases with a low tumor burden, IHC analysis is even more sensitive than certain molecular techniques. ${ }^{22}$ This is another evidence that Sanger sequencing has low sensitivity as compare to some new coming, more sensitive techniques. Therefore, it is now recommended that all patients with HCL must be evaluated for BRAFV600E mutation by using a sensitive molecular assay that can detect the often few $(<10 \%)$ leukemic cells present in the peripheral blood or in diluted bone marrow aspirates. It must be emphasized here that in order to avoid false-negative results, highly sensitive techniques (eg, allele-specific polymerase chain reaction or next-generation sequencing) should be preferred over less sensitive techniques (eg, Sanger sequencing, pyrosequencing, or melting curve analysis) ${ }^{23}$

\section{Conclusion}

Hairy Cell Leukemia is an uncommon lymphoproliferative disorder showing BRAF mutation in a high percentage of HCL cases but identify a minority of HCL cases without BRAF V600E mutation. Therefore BRAF V600E mutation is not $100 \%$ specific for HCL cases but it is still a very useful molecular marker for diagnosis and targeted therapy in patients positive for this mutation.

\section{Acknowledgements}

None.

\section{Conflict of interest}

The author declares no conflict of interest.

\section{References}

1. Polliack A. Hairy cell leukemia: biology, clinical diagnosis, unusua manifestations and associated disorders. Rev Clin Exp Hematol. 2002;6(4):366-388.

2. Bouroncle BA, Wiseman BK, Doan CA. Leukaemic reticuloendotheliosis. Blood. 1958;13(7):609-630.

3. Jones G, Parry-Jones N, Wilkins B, et al. Revised guidelines for the diagnosis and management of hairy cell leukaemia and hairy cell leukaemia variant. Br J Haematol. 2012;156(2):186-195.

4. Kurukulasuriya A, Al-Rashdi A, Al-Muslahi M. Hairy cell leukaemia in oman: four cases. Sultan Qaboos Univ Med J. 2008;8(3):333-338.
5. Dearden C, Else M. Hairy cell leukemia. Curr Oncol Rep. 2016;8(5):337342.

6. Grever MR. How I treat hairy cell leukemia. Blood. 2010;115(1):21-28.

7. Burthem J, Cawley JC. The bone marrow fibrosis of hairy-cell leukemia is caused by the synthesis and assembly of a fibronectin matrix by the hairy cells. Blood. 1994;83(2):497-504.

8. Găman AM. Hairy cell leukemia-a rare type of leukemia A retrospective study on 39patients. Rom J Morphol Embryol. 2013;54(3):575-579.

9. Blombery PA, Wong SQ, Hewitt CA, et al. Detection of BRAF mutations in patients with hairy cell leukemia and related lymphoproliferative disorders. Haematologica. 2012;97(5):780-783.

10. Tiacci E, Trifonov V, Schiavoni G, et al. BRAF mutations in hairy-cell leukemia. N Engl J Med. 2012;364(24):2305-2315.

11. Akarca AU, Shende VH, Ramsay AD, et al. BRAF V600E mutationspecific antibody, a sensitive diagnostic marker revealing minimal residual disease in hairy cell leukaemia. Br J Haematol. 2013;162(6):848-851.

12. Arcaini L, Zibellini S, Boveri E, et al The BRAF V600E mutation in hairy cell leukemia and other mature B-cell neoplasms. Blood. 2012;119(1):188-191.

13. Jan A Burger. BRAF mutation: supporting diversity in HCL. Blood. 2012;119(14):3193-3194.

14. Xi L, Arons E, Navarro W, et al. Both variant and IGHV4-34expressing hairy cell leukemia lack the BRAF V600E mutation. Blood. 2012;119(14):3330-3332.

15. Dietrich $\mathrm{S}$, Glimm $\mathrm{H}$, Andrulis $\mathrm{M}$, et al. BRAF inhibition in refractory hairy-cell leukemia. N Engl J Med. 2012;366(21):2038-2040.

16. Tiacci E, Park JH, De Carolis L, et al. Targeting mutant BRAF in relapsed or refractory hairy-cell leukemia. $N$ Engl J Med. 2015;373(18):17331747.

17. Grever MR, Abdel-Wahab O, Andritsos LA, et al. Consensus guidelines for the diagnosis and management of patients with classic hairy cell leukemia. Blood. 2017;129(5):553-560.

18. Robak T, Matutes E, Catovsky D, et al. Hairy cell leukaemia: ESMO clinical practice guidelines for diagnosis, treatment and follow-up. Ann Oncol. 2015;26(Suppl 5):v100-107.

19. Boyd EM, Bench AJ, Vant Veer MB, et al. High resolution melting analysis for detection of BRAF exon 15 mutations in hairy cell leukaemia and other lymphoid malignancies. Br J Haematol. 2011;155(5):609-612.

20. Tschernitz S, Flossbach L, Bonengel $\mathrm{M}$, et al. Alternative BRAF mutations in BRAF V600E-negative hairy cell leukaemias. $\mathrm{Br} J$ Haematol. 2014;165(4):529-533.

21. Wang XJ, Kim A, Li S. Immunohistochemical analysis using a BRAF V600E mutation specific antibody is highly sensitive and specific for the diagnosis of hairy cell leukemia. Int J Clin Exp Pathol. 2014;7(7):43234328 .

22. Andrulis M, Penzel R, Weichert W, et al. Application of a BRAF V600E mutation-specific antibody for the diagnosis of hairy cell leukemia. Am J Surg Pathol. 2012;36(12):1796-1800.

23. Tiacci E, Schiavoni G, Forconi F, et al. Simple genetic diagnosis of hairy cell leukemia by sensitive detection of the BRAF-V600E mutation. Blood. 2012;119(1):192-195. 